Military Technical College Kobry El-Kobbah, Cairo, Egypt

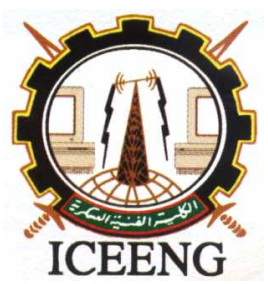

\author{
$6^{\text {th }}$ International Conference \\ on Electrical Engineering \\ ICEENG 2008
}

\title{
Real-Time physical dynamic simulations of electric machine drives with hardware-in-the-loop
}

\author{
By \\ O. A. Mohammed \\ S. Liu* \\ Z. Liu* \\ N. Y. Abed ${ }^{*}$
}

\section{Abstract:}

This paper presents the real time simulation of electric machine control and drive by using hardware-in-the-loop (HIL) simulation technology. In our research implementations, two simulation loops for the control and drive of the same machine are built. One simulation loop is formed using the actual machine. Another simulation loop is formed using a physics-based phase variable model of the same machine. The physics-based phase variable model of the machine is developed from the solutions of nonlinear transient Finite Element (FE) computations covering a complete ac cycle. The developed physics-based base model includes both the time and space harmonics representing the effects of rotor position, slotting, and nonlinear magnetization of the core, etc. The hardware-in-the-loop simulation of the machine drives provides an experimental environment for examining the machine model and studying the control algorithm. A 2-hp 6-pole PM synchronous machine is used to present the physics-based phase variable modeling; a 10-pole PM machine is used to demonstrate the hardware-inthe-loop simulation with the actual machine and its physics-based model.

\section{Keywords:}

Hardware-in-the-loop simulation, machine control and drive, machine model, field computations. 


\section{Introduction:}

This paper presents the utilization of the hardware-in-the-loop simulation for machine control applications. Research was carried out in the HIL simulation of machine control and drive utilizing the actual machine. Also, studies were performed to realize the HIL simulation of machine control and drive using the actual machine and the machine model at the same time. The main purpose of this study is building a machine model with high level of accuracy and implementing it to the controller design in a hardware-in-the-loop simulation environment.

In this paper, first, the details of the RTLab real time hardware-in-the-loop simulation system for motor control and drive applications are described. Then the physics-based phase variable modeling of PM synchronous machine is reviewed, including the equation, parameter determination, Simulink implementation, and verification. Finally, the hardware-in-the-loop simulation of PM machines is presented with two simulation loops operating simultaneously; one loop uses the actual machine and the other loop uses the developed physics-based phase variable model.

\section{RTLab real time hardware-in-the-loop system description:}

The overall system architecture is shown in Figure 1. The system consists of a drive board, target machines, the master machine, and the motor coupling system.

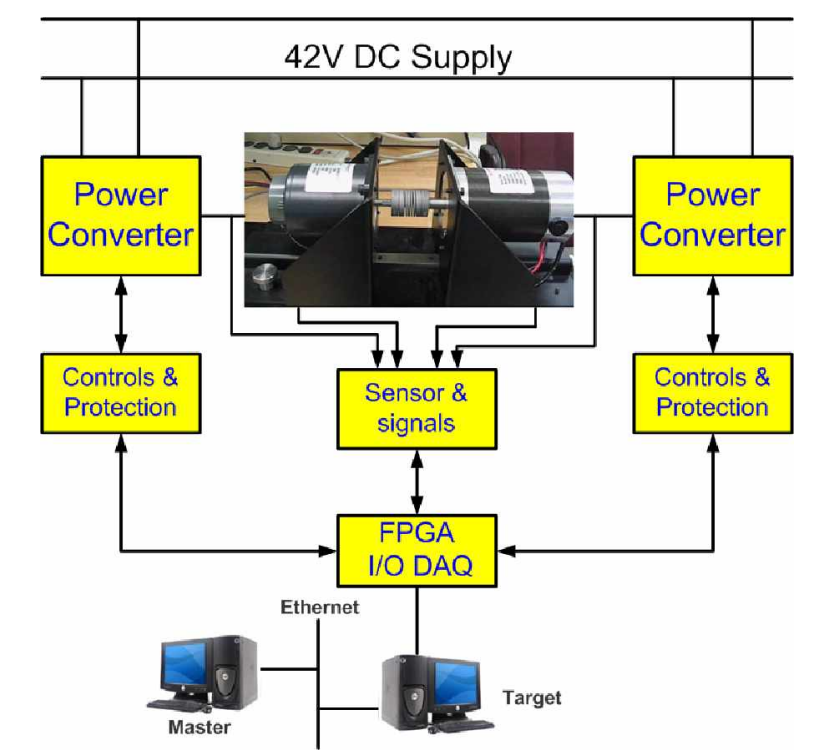

Figure(1): The real time simulator structure 


\subsection{The Drive Board}

Figure 2 shows the drive board layout. The drive board consists of two independent three phase inverters. Each 3-phase inverter uses MOSFETs as switching devices. The drive board is used to perform a variety of control experiments on both AC and DC machines. The main features of the drive board are:

- Two independent 3-phase PWM inverters for complete simultaneous control of two machines at the same time.

- Digital PWM input channels for real-time digital control

- Complete digital/analog interface with the FPGA DAQ card.

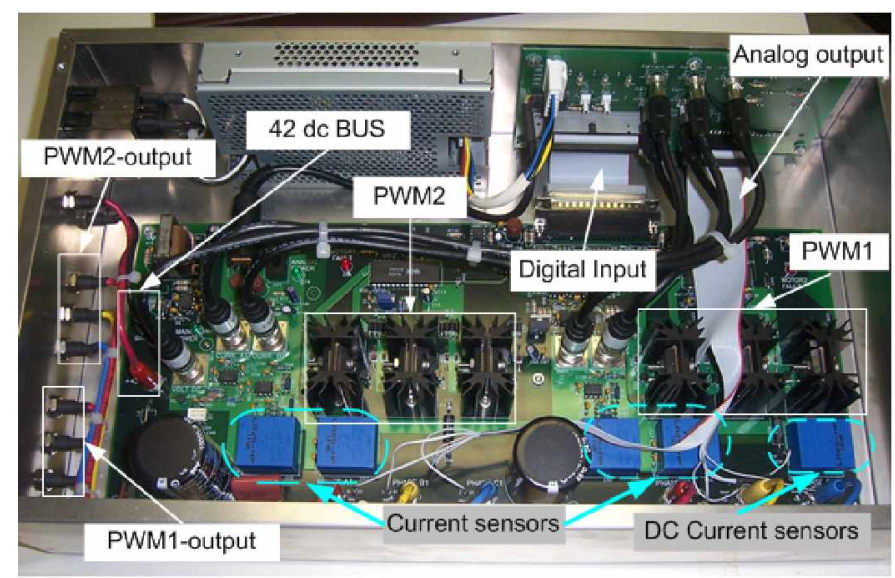

Figure(2): Electric drive board layout

The drive board is protected by an over current relay for each inverter. The relay fault status can be cleared by using hardware switch on the board or through a control signal from the command station. The power supply for the inverters 3-phase bridge drivers is derived from the DC Bus through a converter. Sensors are used to measure the output current of the inverter. Only " $a$ " and " $b$ " phase currents are sensed. The sensors are used to measure the inverter output voltages and the dc bus voltage.

\subsection{Block Diagram and Schematic Interface}

The real-time simulator is based on real-time, distributed simulation platform. It is optimized to run Simulink and SimPowerSystems in real-time, with fixed time step solvers, on a PC-Cluster.

MATLAB is widely used in the dynamic system simulation and control systems analysis. MATLAB is a numerical analysis programming shell with add-on components called toolboxes. Simulink, a toolbox of MATLAB, is dynamic system simulation software that provides a convenient graphical user interface for building system models based on their equations. 
The Real Time Workshop (RTW) toolbox is capable of generating real time code for Simulink models [1]. The real-time code is used to control a system in the HIL environment. The data generated by any of the system components, digital or hardware, can be displayed in real-time and/or saved for later use.

The system uses the Simulink as an interface for building, editing and viewing graphic models in block-diagram format. The block diagram models are converted into C-code using RTW, and then transferred in to target machine for real-time simulation. Figure 3 shows the real time simulator software subsystems architecture.

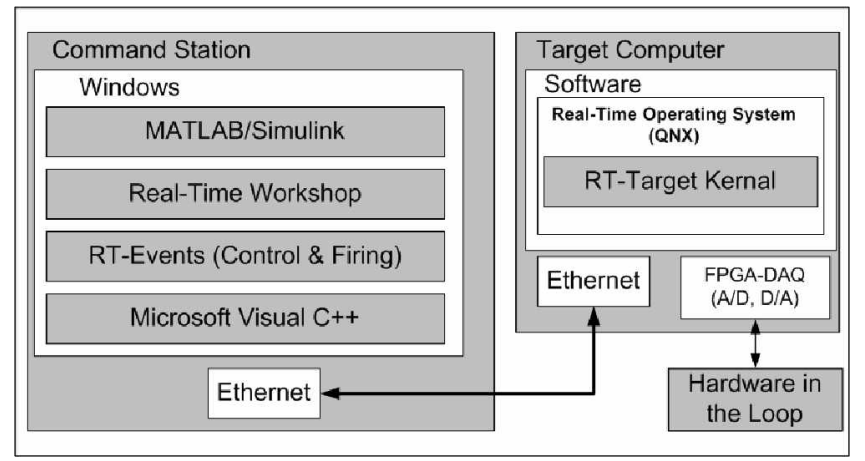

Figure(3): Host-target real time software architecture

\subsection{Inputs and Outputs $(\mathrm{I} / \mathrm{O})$}

A major requirement for real-time HIL applications is the interfacing with real world hardware devices, controller or a physical plant. The DAQ (I/O) provides the interface between the target computer and the HIL system. High performance I/O Xilink Virtex-II pro FPGA DAQ was used.

This FPGA DAQ reads the analog data collected from the controlled inverter-motor set and generate the digital control signals for the two PWM inverters at time step as low as 10 microseconds according to the implemented control algorithm.

In the real-time simulator, I/O interfaces are configured through Simulink custom blocks. The inputs and outputs interface to these blocks can be managed through Simulink, without writing low-level driver codes.

The RT shell generates automatically the I/O drivers and models code that handle and direct the flow data from or into the physical I/O cards. 


\subsection{Simulator Configuration}

The configuration of the real time simulator consists of following integrated subsystems:

- One or more target computation nodes (PC's) with real time operating system (e.g. QNX real-time operating system or Linux).

- Command or host station that represents the user interface with the system through which the user can control and change the system parameters. It also, coordinates the simulations between the targets, collects the results for display, and control the communication between the command station and the targets as well as the communication between the target PC's.

- I/O's interface with the simulator (analog I/O, digital I/O, PWM I/O, encoders, etc). I/O's can be managed by dedicated processors.

The simulator transfers data and control signals between the host and the target PC through an Ethernet connection. This network connection allows the distribution of model computation between the system nodes and thus achieves small simulation time step size. Full control of the target application and computer using Simulink is provided to the user through the RT-shell.

RT-EVENTS are used to generate IGBT inverter signals (Pulses) for real-time simulation of inverters in the DC-stage $[2,3]$.

\section{HIL simulation using the actual machine and the physics-based phase variable model simultaneously}

\subsection{The physics-based phase variable model $[4,5]$}

\subsubsection{Equations}

The voltage, flux linkage, torque, and the motion equations of the developed physicsbased phase variable model are given by:

$$
\begin{aligned}
& V_{a b c}=r_{a b c} i_{a b c}+d \psi_{a b c}(\theta) / d t \\
& \psi_{a b c}(\theta)=\psi_{s a b c}(\theta)+\psi_{r a b c}(\theta) \\
& \Psi_{s a b c}(\theta)=L_{a b c}(\theta) i_{a b c} \\
& T_{m}=p\left(\frac{1}{2} i_{a b c}^{T} \cdot \frac{d L_{a b c}(\theta)}{d \theta} \cdot i_{a b c}+i_{a b c}^{T} \cdot \frac{d \psi_{r a b c}(\theta)}{d \vartheta}\right)+T_{c o g}(\theta) \\
& J \cdot d \omega / d t=T_{m}-B \omega-T_{L} \\
& d \theta / d t=\omega
\end{aligned}
$$


Where:

$V_{a b c}$ : voltage matrix, $V_{a b c}=\left[\begin{array}{lll}V_{a} & V_{b} & V_{c}\end{array}\right]^{T}$.

$i_{a b c}$ : current matrix, $i_{a b c}=\left[\begin{array}{lll}i_{a} & i_{b} & i_{c}\end{array}\right]^{T}$.

$r_{a b c}$ : resistance matrix, $r_{a b c}=\left[\begin{array}{lll}r_{a} & r_{b} & r_{c}\end{array}\right]$.

$\theta$ : rotation angle/rotor position.

$\psi_{a b c}(\theta)$ : flux linkage matrix, $\psi_{a b c}(\theta)=\left[\begin{array}{lll}\psi_{a}(\theta) & \psi_{b}(\theta) & \psi_{c}(\theta)\end{array}\right]^{T}$.

$\psi_{\text {sabc }}(\theta)$ : flux linkage contributed by winding currents,

$$
\psi_{s a b c}(\theta)=\left[\begin{array}{lll}
\psi_{s a}(\theta) & \psi_{s b}(\theta) & \psi_{s c}(\theta)
\end{array}\right]^{T} .
$$

$\psi_{\text {rabc }}(\theta)$ : flux linkage contributed by permanent magnets,

$$
\psi_{r a b c}(\theta)=\left[\begin{array}{lll}
\psi_{r a}(\theta) & \psi_{r b}(\theta) & \psi_{r c}(\theta)
\end{array}\right]^{T} .
$$

$T_{m}$ : output torque.

$p$ : number of pole pairs.

$T_{\operatorname{cog}}(\theta)$ : cogging torque.

$J$ : inertia.

$\omega$ : angular speed.

$B$ : friction factor.

$T_{L}$ : load torque.

$L_{a b c}(\theta)$ : inductance matrix of stator winding described as:

$$
L_{a b c}(\theta)=\left[\begin{array}{lll}
L_{a a}(\theta) & L_{a b}(\theta) & L_{a c}(\theta) \\
L_{b a}(\theta) & L_{b b}(\theta) & L_{b c}(\theta) \\
L_{c a}(\theta) & L_{c b}(\theta) & L_{c c}(\theta)
\end{array}\right]
$$

Due to the nonlinear magnetization of the core, the stator winding flux linkage $\psi_{a b c}$ and the inductance matrix $L_{a b c}$ of surface mounted PM machines are theoretically function of the winding current and the rotor position. Since the magnetic field of PM machines is dominated by the permanent magnets, $\psi_{a b c}$ and $L_{a b c}$ are considered to be the function of the rotor position only in the developed physics-based model.

$L_{a b c}(\theta), \psi_{r a b c}(\theta)$, and $T_{c o g}(\theta)$ are determined from the solutions of the nonlinear transient FE computation covering a complete ac cycle. The utilization of $L_{a b c}(\theta)$, $\psi_{\text {rabc }}(\theta)$, and $T_{\text {cog }}(\theta)$ obtained from FE solutions enables the machine model expressed by equations (1) through (6) include the effect of the rotor position, the slotting, and the associated nonlinear magnetization variation of the core. 


\subsubsection{Inductance, flux linkage, and cogging torque determination}

A 6-pole, 36-slot, 2hp surface mounted PM synchronous machine is used as an example machine to explain the implementation of the proposed physics-based phase variable model.

Figures. 4-6 show the inductance $L_{a b c}(\theta)$, flux linkage due to permanent magnet $\psi_{\text {rabc }}(\theta)$, and the cogging torque $T_{\text {cog }}(\theta)$, respectively, obtained from the steady state solutions of nonlinear transient FE computation covering complete ac cycles. The core magnetization variation due to the rotation is automatically included owing to the nonlinear FE computation.

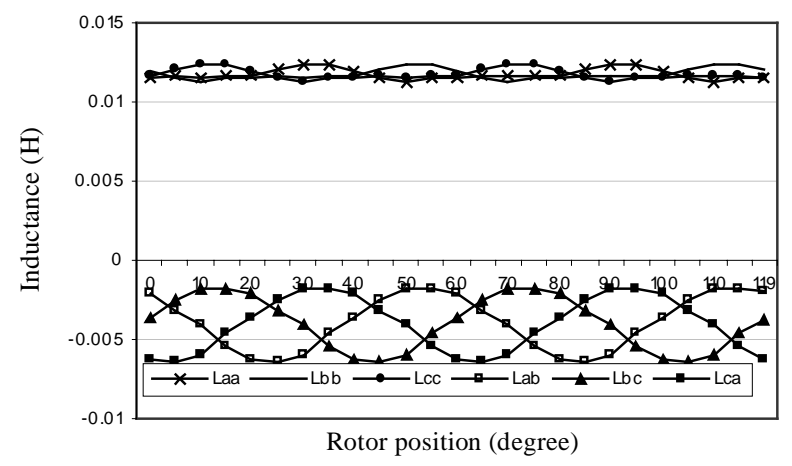

Figure(4): Rotor position dependency of inductance

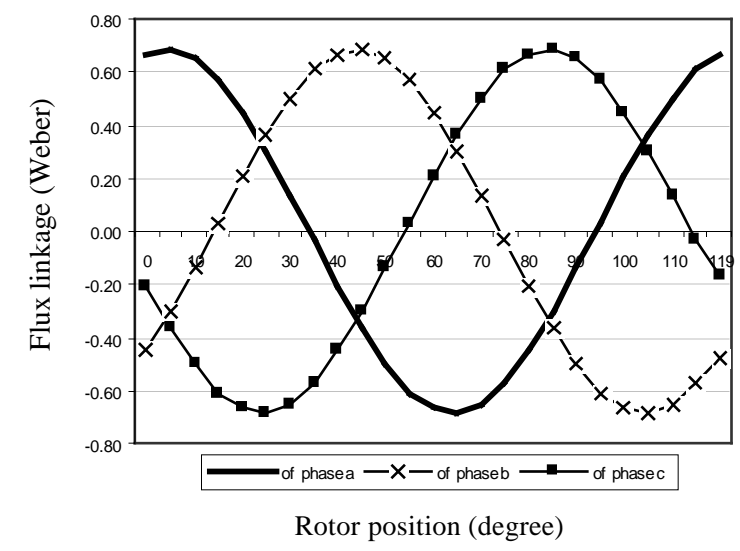

Figure(5): Rotor position dependency of flux linkage due to PM 


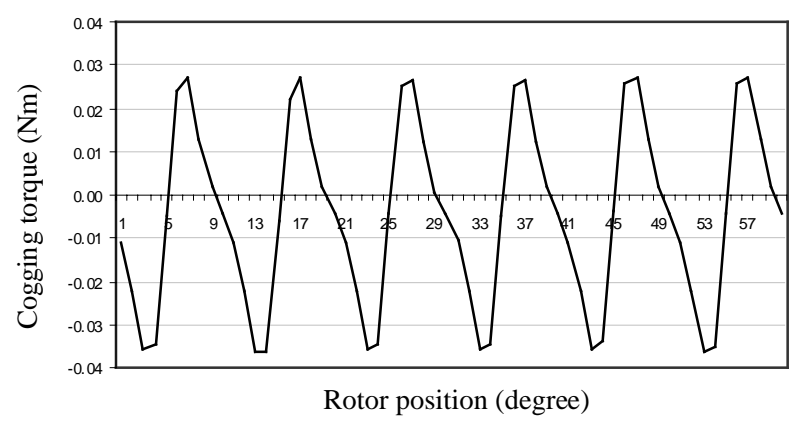

Figure(6): Cogging torque

\subsubsection{Simulink implementation}

Two approaches can be used to develop the machine block in Simulink according to (1) to (6). One approach is the equation-based method; the other one is the circuit component-based method. By the equation-based method, the machine model is built according to the mathematical expression of the winding circuits [4]. By the circuitcomponent-based method, the machine model is built based on the circuit description of the machine winding [5].

\subsubsection{Verification by comparison with $\mathrm{FE}$ modeling}

A FE machine block is obtained by exporting the 2D nonlinear transient FE description of the PM motor to Simulink. The mesh of the FE machine block is kept the same as the mesh used for the inductance, flux linkage, as well as the cogging torque calculations for building the physics-based phase variable model.

Figure 7 shows the machine block built with the FE description of the machine. The input of the FE machine block is currents, which are obtained using three current measurement blocks (CM). The back EMF is calculated by performing the timederivative operation on the flux linkage due to the permanent magnets. The controlled voltage sources (CVS) and the current measurement blocks, at the left side of Figure 7, are used to connect the FE machine block with the external circuits

Figure 8 shows the speed control system used for examining the various types of machine models. In order to investigate the intrinsic characteristic of the physics-based phase variable model and the full FE model, a filter circuit is added to remove the impacts of PWM on the torque, voltage and current profiles. 


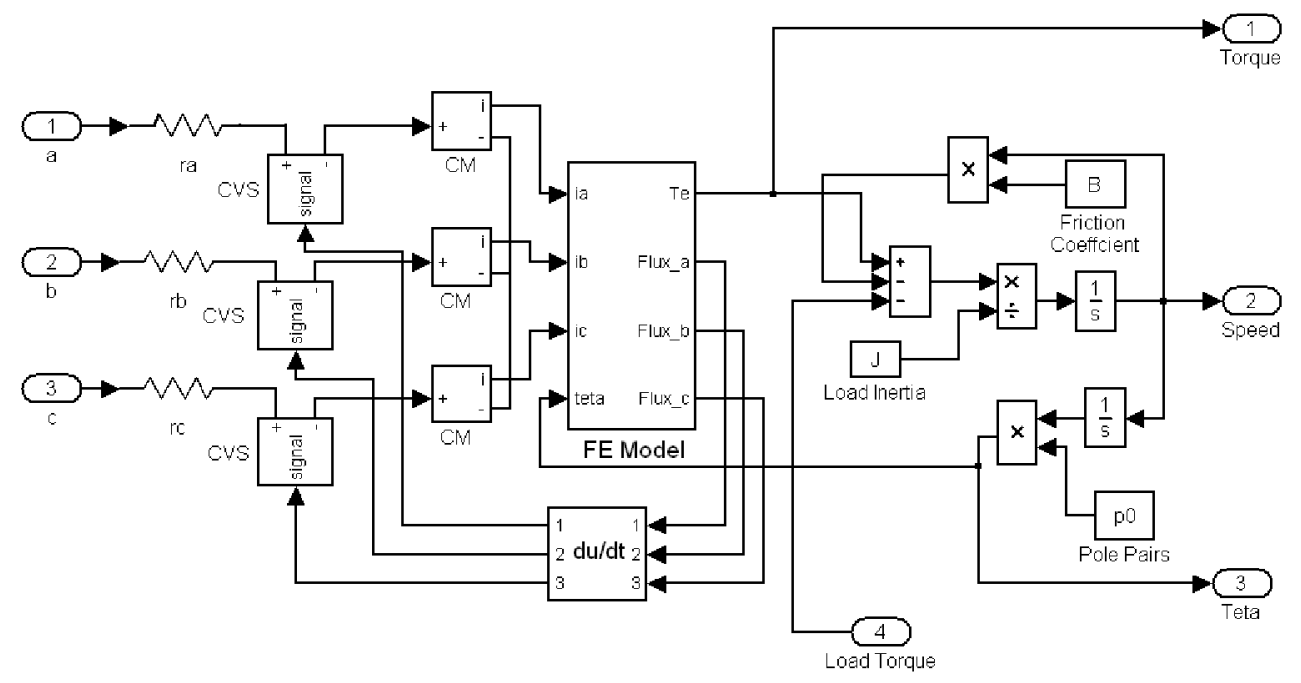

Figure(7): The FE motor model

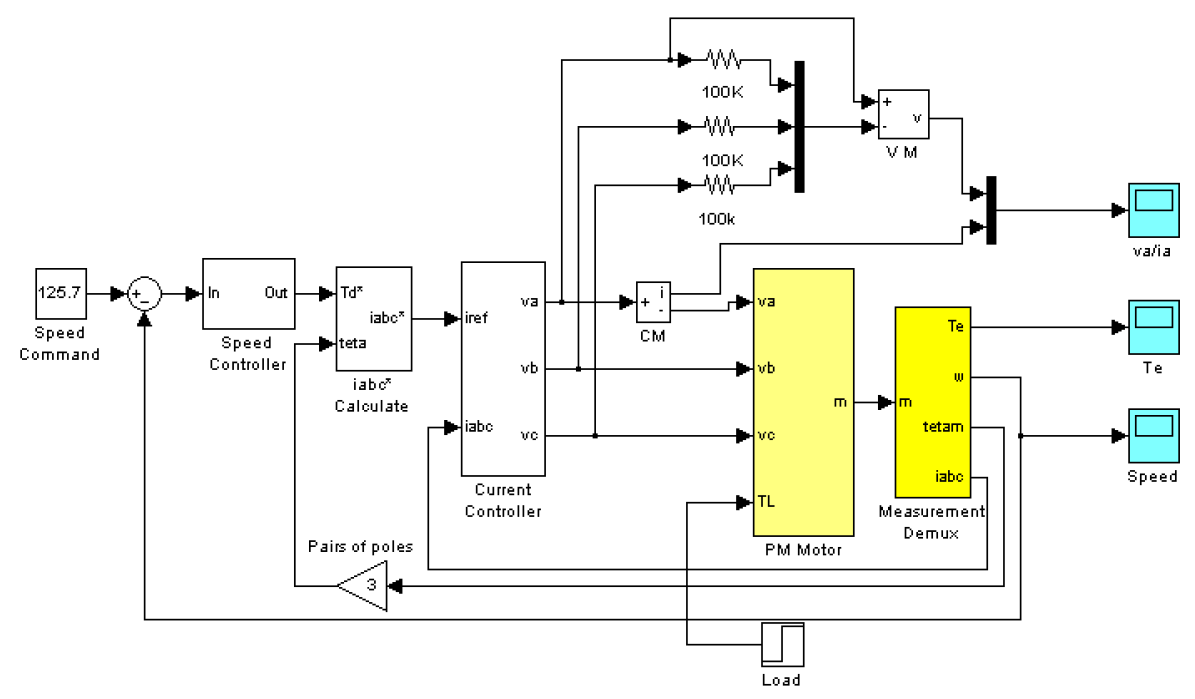

Figure(8): PM motor vector control system

Figure 9 show the obtained torque profiles during the starting process of the PM machine. The torque obtained by using the developed physics-based phase variable model, see in Figure 9(a), is very close to the torque profile obtained by using the FE model, seen in Figure 9(b).

Figure 10 shows the voltage and current profiles of phase " $a$ " under the steady state operation condition. It can be seen that the voltage and current waveforms obtained by using the developed physics-based phase variable model and the FE model are very close. Both of them show harmonic components, which do not appear in the voltage and current waveforms obtained by using the conventional dq-model. 



Figure(9): Torque profile obtained by using (a) physics-based phase variable model,(b) the FE model

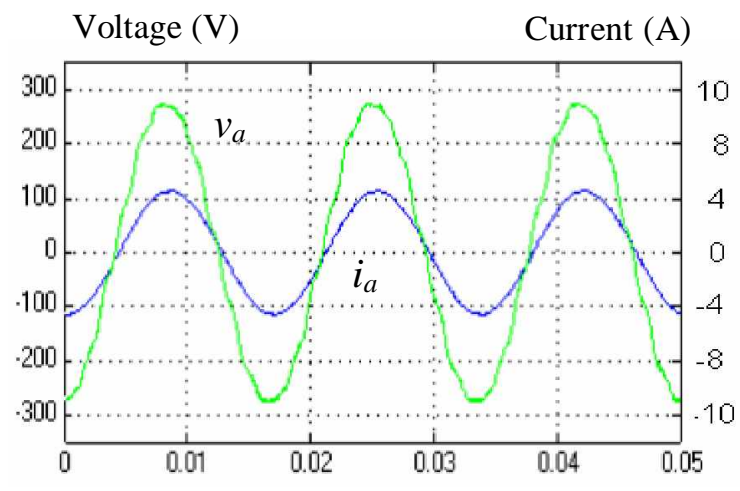

(a)

Time (s)

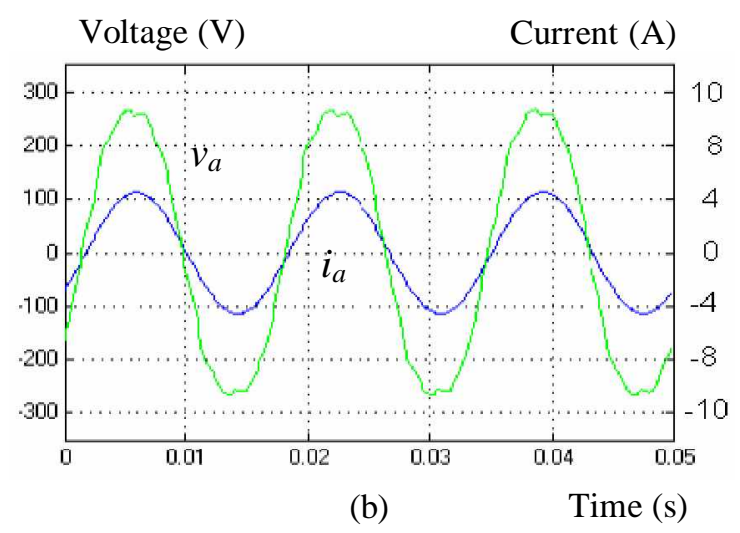

Figure(10): Voltage and current profiles obtained by using (a) physics-based phase variable model, (b) the FE model 


\subsection{HIL simulation using the actual machine and the physics-based phase variable model simultaneously}

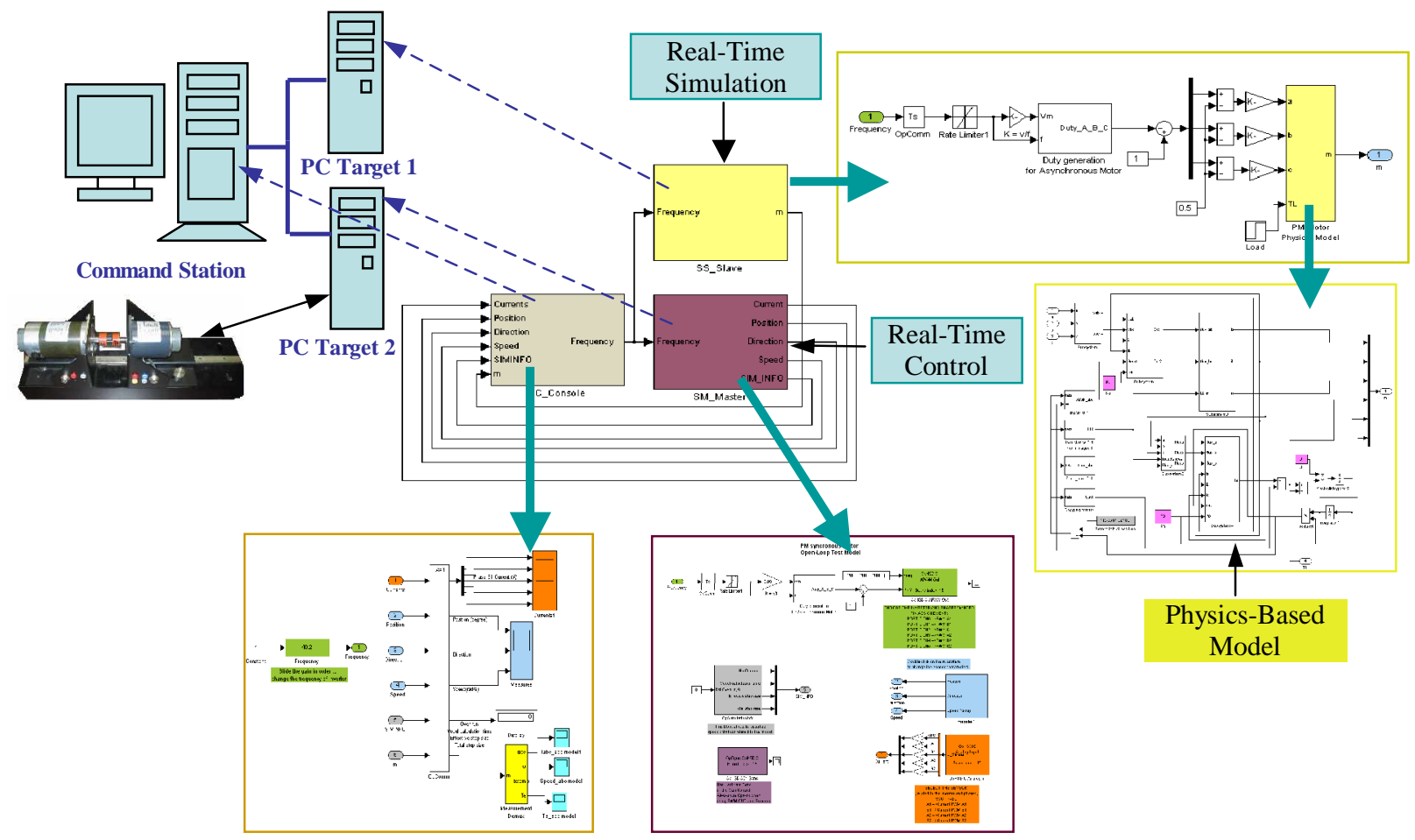

Figure (11): Diagram of the experimental system used for the examination of the developed physics-based phase variable model

RTLab simulation environment allows the real-time simulation of the machine drive using the machine model and the real time control on the actual machine to be run simultaneously at the same time step. Figure 11 shows the diagram of the experimental system. Target computer 1 is used to perform the simulation of the machine drive using the developed physics-based phase variable model. Target computer 2 is used to perform the real time control of the actual machine.

A 10 pole PM synchronous machine was used to perform the experiment shown in Figure 11. The physics-based phase variable model of the 10 pole PM machine was developed using the approach explained in the preceding section. The developed physics-based phase variable model and the circuit model of the drive board were assigned to target computer 1 to perform the real time simulation. The real time control of the actual 10-pole PM machine was assigned to target computer 2. The V/f control approach is used in both the real time simulation of the drive and the real time control of the actual machine. Figure 12 shows the obtained result. 
In Figure 12, from the top to the bottom is the DC-bus voltage, the currents measured at the actual machine, and the currents obtained using the developed physics-based machine model. The agreement shown in the current waveforms indicates that the developed physics-based phase variable model can provide the expected representation of the actual machine in a simulation environment.

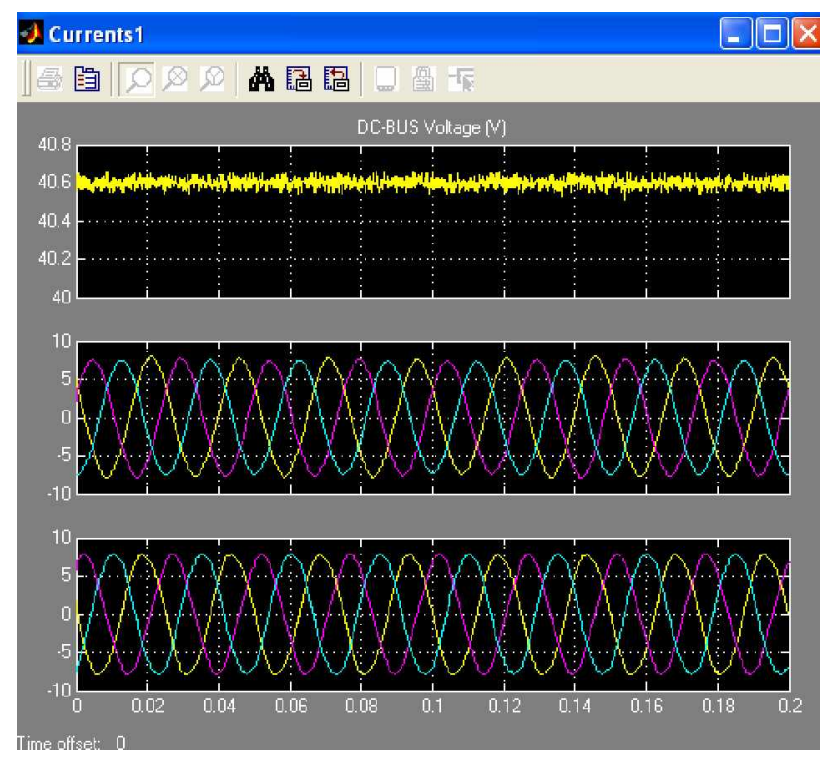

Figure (12): Result for the experimental examination of the physics-based phase variable model of a 10 pole PM machine

\section{Conclusions:}

This paper presented the physics-based phase variable model of PM synchronous machines and the simultaneous real time simulation of the machine control in conjunction with the real time control of the actual machine. The RTLab hardware-inthe-loop real time simulation environment was used for implementing the proposed idea. The results, obtained from the model verification and the hardware-in-the-loop simulation experiments indicate that the developed physics-based phase variable model describes the machine with high accuracy. The next step of our research work will be on developing an equivalent hardware representation for the actual machine utilizing the physics-based phase variable model. The expected result will facilitate the controller studies before the actual fabrication of machines and their driving controllers. 


\section{References:}

[1] S. Abourida, C. Dufour, J. Belanger, "Real time and Hardware in the Loop Simulation of Electric Drives and Power Electronics: Process, problems and solutions," International conference on power Electronics 2005.

[2] Opal-RT Technologies, RT-Lab User's Guide ,2006.

[3] RT-Events, Version 2.2 User Guide

[4] Mohammed, S. Liu, and Z. Liu, "Physical modeling of pm synchronous motors for integrated coupling with machine drives," IEEE Transactions on Magnetics, Vol. 41, No. 5, May 2005, pp. 1628-1631.

[5] Mohammed, S. Liu, and Z. Liu, "Phase variable model of PM synchronous machines for integrated motor drives," IEE Proceedings Science, Measurement \& Technology, vol.151, no. 6, pp.423-429, Nov. 2004. 